\title{
Thermodynamics of mixing in rutile-stishovite solid solution from quantum-mechanical calculations
}

\author{
Victor L. Vinograd, Daniel J. Wilson and BjörnWinkler \\ Institute of Geosciences, University of Frankfurt, Frankfurt a. M., Germany
}

The occurrence of Si-rich rutile $\left(18 \mathrm{~mol} \%\right.$ of $\left.\mathrm{SiO}_{2}\right)$ in a chromitite body within the ultra-high-pressure metamorphic sequence of Yarlunzangbu ophiolite in Southern Tibet (Yang et al. 2003) suggests that under certain conditions rutile forms a solid solution with stishovite. This opens the possibility of using the stishovite-coesite equilibrium for estimating pressures of crystallization of rocks containing coesite and rutile, since rutile can be treated as stishovite diluted with $\mathrm{TiO}_{2}$. The association of rutile and coesite is typical for eclogite-type inclusions in diamonds (e.g. Kaminsky et al., 2000). The $\mathrm{SiO}_{2}$ content of rutiles in coesite bearing rocks has yet not been studied systematically. A recent experimental study of Ren et al. (2005) showed, however, that at $10 \mathrm{GPa}$ and $2073 \mathrm{~K}$ rutile in association with coesite dissolved about 2 mole $\%$ of $\mathrm{SiO}_{2}$. This concentration level is detectable with a variety of analytical techniques. To calculate the rutilecoesite equilibrium as a function of pressure and temperature one needs the thermodynamic properties of pure coesite and stishovite and a model for the activity of stishovite in the stishovite-rutile solid solution:

$$
\Delta G_{T, P}^{\mathrm{Stish}}+R T \ln a_{\mathrm{SiO}_{2}}^{\mathrm{Stish}}=\Delta G_{T, P}^{\mathrm{Coes}}
$$

Here the thermodynamic mixing properties of the $\mathrm{SiO}_{2}$ $\mathrm{TiO}_{2}$ solid solution are modeled with the recently developed Double Defect Method (DDM) (Vinograd et al., 2008). The DDM is based on first principles calculations of the formation energies of supercell structures with single B- and A- and paired BB- and AA-type defects inserted at all possible distances in the supercells of A and B end-members. These energies are converted to the so-called pair interaction energies, $J_{n}$, with the equation

$$
J_{n}=\left(2 \Delta H_{\text {single }}-\Delta H_{\text {double }}^{(n)}\right) / D_{n},
$$

where $\Delta H_{\text {single }}$ and $\Delta H_{\text {double }}^{(n)}$ are the excess enthalpies of the structures with single and double defects and $D_{n}$ is the degeneracy factor for the pair of defects at the $n$ th distance. These $J_{n}$ values are further used to get an approximation for the excess enthalpy of any $\mathrm{A}_{x} \mathrm{~B}_{(1-x)}$ structure within the supercell using the equation

$$
\Delta H_{i}=\sum_{n} f_{\mathrm{AB}}^{(n)} J_{n}
$$

where $f_{\mathrm{AB}}^{(n)}$ is the frequency of pairs of AB-type at the $n$-th distance within the supercell. Such an approximation makes it possible to use a Monte Carlo algorithm to calculate temperature dependent enthalpies of mixing within a much bigger supercell, where thermodynamic properties approach those of a real crystal. The Gibbs free energies of mixing and the thermodynamic activities can then be calculated from the temperature-dependent enthalpies with the thermodynamic integration method (Warren et al., 2001).

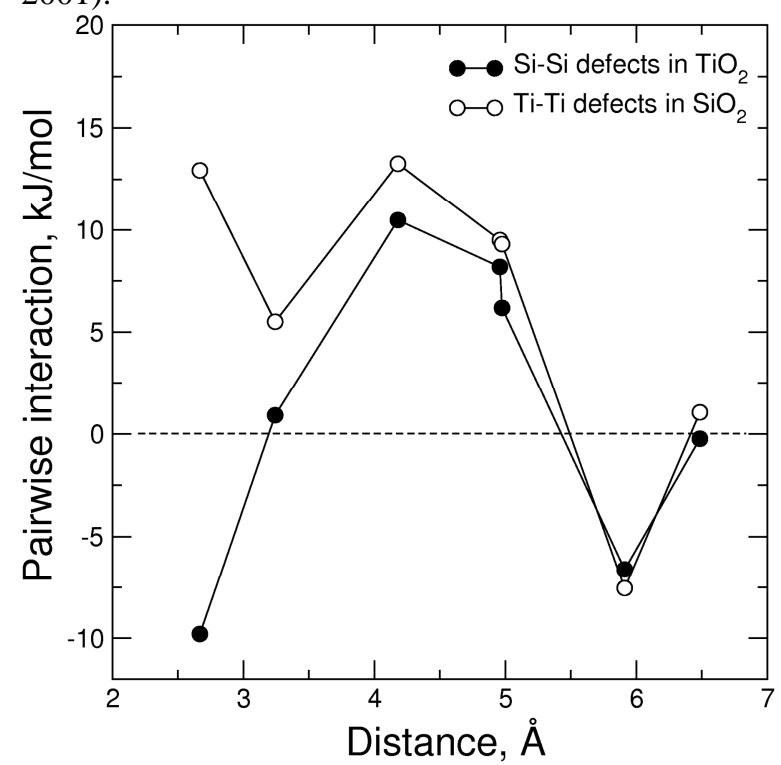

Fig. 1. The effective pair interactions, the $J \mathrm{~s}$, at $3 \mathrm{GPa}$ in the stishovite-rutile solid solution calculated with the DDM. Positive sign corresponds to the clustering tendency.

In this study we have considered $2 \times 2 \times 3$ supercells of $P 4 / \mathrm{mnm}$ stishovite and rutile with the parameters $(\boldsymbol{a}=$ $8.3602 \AA$ and $c=8.004 \AA$ ). The size of these supercells permits consideration of seven SiSi- and TiTi-type defects in rutile and stishovite, respectively, located at different distances within the range of 2.7-6.5 $\AA$. The total energies of the end-members and of the structures with the double defects were calculated with the density functional theory (DFT) with the PBE exchange-correlation functional. The calculations were performed with the CASTEP code (Clark et al., 2005) at the Centre for Scientific Computing at the University of Frankfurt. The calculation used a plane-wave basis set cut-off of $380 \mathrm{eV}$ and a $4 \times 4 \times 4$ Monkhorts-Pack grid of k-points. Ultrasoft pseudopotentials (Kresse and Hafner, 1994) were used to represent the effective nonlocal potential due to the nucleus and core-electrons. These settings permitted the total energy to be determined with an accuracy of $0.01 \mathrm{eV}$ per supercell. The results of these calculations at the pressures of 3 and $8 \mathrm{GPa}$ are listed in Table 1 and plotted in Figure 1. 
Table 1 shows that the excess energies of both SiSi and TiTi defects are large and positive. This means that the solid solution has a strong tendency to phase separate. However, the excess energies of the SiSi defects are significantly smaller than those of the TiTi defects. This suggests that a certain range of Si-substitution is possible at the rutile side. It is also clear that the mixing effects are insensitive to pressure changes in the range of 3-8 GPa.

Table 1. The excess energies of single and double defect structures (in $\mathrm{eV}$ per supercell) in rutile and stishovite: $2 \times 2 \times 3$ supercell, 24 exchangeable sites.

\begin{tabular}{|c|c|c|c|c|c|}
\hline \multicolumn{5}{|c|}{ Single defects } \\
\cline { 1 - 4 } $3 \mathrm{GPa}$ & \multicolumn{2}{c|}{$8 \mathrm{GPa}$} & \\
\\
\hline \multicolumn{2}{|c|}{$\mathrm{Si}$} & $\mathrm{Ti}$ & $\mathrm{Si}$ & $\mathrm{Ti}$ & \\
\hline \\
\hline \multicolumn{6}{|c}{ Double defects } \\
\hline \multirow{2}{*}{ Dist., $\AA$} & 1.27 & \multirow{2}{*}{$D_{n}$} & \multicolumn{2}{|c|}{$3 \mathrm{GPa}$} & \multicolumn{2}{c}{$8 \mathrm{GPa}$} \\
\cline { 3 - 5 } & & $\mathrm{SiSi}$ & $\mathrm{TiTi}$ & $\mathrm{SiSi}$ & $\mathrm{TiTi}$ \\
\hline 2.67 & 1 & 1.42 & 2.36 & 1.41 & 2.33 \\
\hline 3.24 & 1 & 1.30 & 2.38 & 1.29 & 2.37 \\
\hline 4.18 & 2 & 1.10 & 2.22 & 1.07 & 2.20 \\
\hline 4.96 & 2 & 1.15 & 2.30 & 1.12 & 2.28 \\
\hline 4.97 & 2 & 1.19 & 2.30 & 1.17 & 2.29 \\
\hline 5.91 & 4 & 1.59 & 2.81 & 1.60 & 2.82 \\
\hline 6.49 & 4 & 1.33 & 2.45 & 1.33 & 2.43 \\
\hline
\end{tabular}

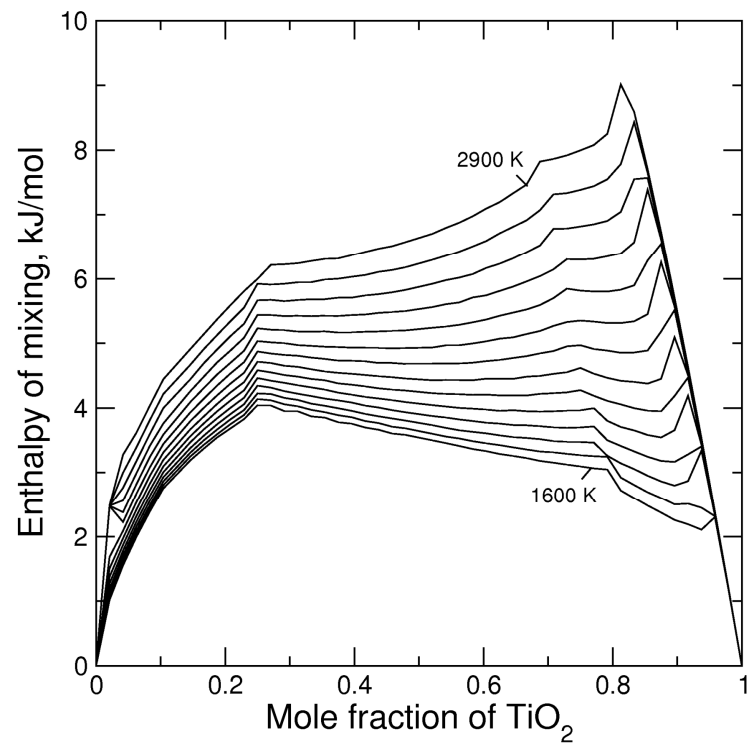

Fig. 2. The isotherms of the enthalpy of mixing in the rutilestishovite solid solution from Monte Carlo simulations.

Figure 1 further shows that the pair interaction energies which correspond to $\mathrm{SiSi}$ defects in rutile are negative at the shortest distance, what means that such defects would tend to dissociate within the rutile structure, forming $\mathrm{Si}-\mathrm{Ti}$ pairs and thus favouring mixing.

The $J_{n}$ at intermediate compositions were linearly interpolated between the values which correspond to $\mathrm{SiSi}$ and TiTi defects. The combination of the effective pair interactions according to Equation 3 permits thus evaluation of the excess enthalpy of any configuration in the supercell. This equation was further used within a Monte Carlo algorithm to calculate the temperature dependent enthalpies of mixing. Monte Carlo simulations were performed with a $12 \times 12 \times 12$ supercell containing 5184 exchangeable sites. The calculations were done in the temperature range of 1600-2900 K with steps of $100 \mathrm{~K}$ and 0.02083 in the temperature and composition, respectively. The averages were calculated over the last half of $6 \cdot 10^{7}$ Monte Carlo steps. The calculated temperature-dependent enthalpies and the free energies are shown in Figures 2 and 3, respectively. Figure 3 shows that the mixing is restricted to Ti-rich compositions. The $\mathrm{SiO}_{2}$ concentration in rutile in coexistence with stishovite progressively increases with the temperature and reaches about $10 \mathrm{~mol} \%$ at $2900 \mathrm{~K}$. The nearly flat segments in the enthalpy of mixing isotherms in Fig. 2 are a consequence of the phase separation which occurred in the course of the Monte Carlo simulations. It is observed that the enthalpy of mixing isotherms at the diluted concentrations follow the ideal mixing regime. This becomes apparent by comparing the enthalpy and the entropy isotherms, (Fig. 4). The ideal mixing becomes favourable due to the very strong tendency to phase separation which leads to the formation of the two phases with the Si- and Ti-rich compositions. The minorities of $\mathrm{Si}$ and $\mathrm{Ti}$ atoms are dispersed within the Ti- and Si-rich matrixes producing large entropy.

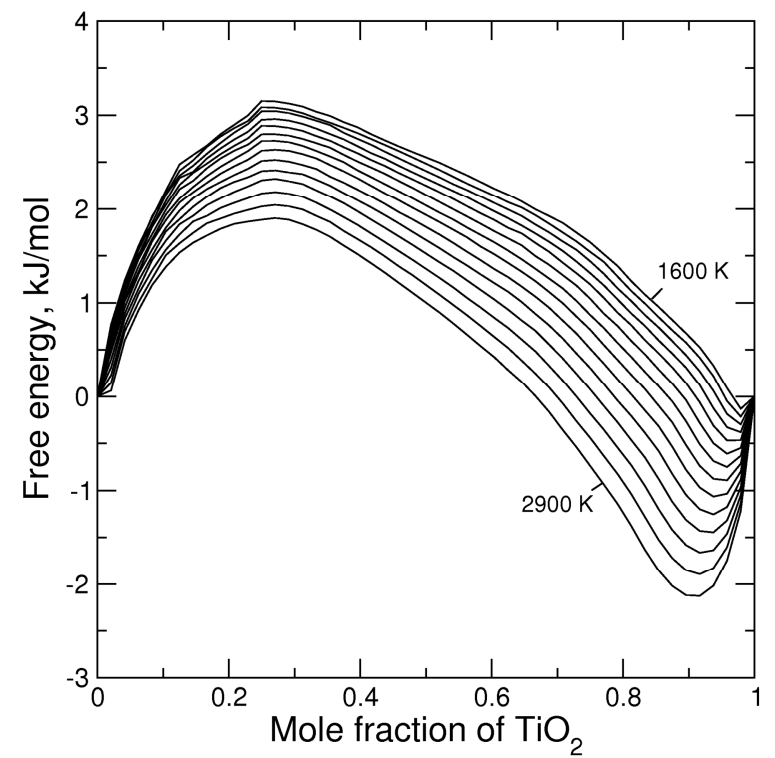

Fig. 3. The free energy of mixing calculated from the Monte Carlo results with the method of thermodynamic integration.

The excess free energies of mixing were fitted with the Redlich-Kister polynomial

$$
G_{\text {excess }}=x_{1} x_{2} \sum_{i=1}^{n}\left(A_{n}+B_{n} T+C_{n} T^{2}\right)\left(x_{2}-x_{1}\right)^{n}
$$

where $x_{1}$ and $x_{2}$ are the mole fractions of stishovite and rutile. The values of $A, B$ and $\mathrm{C}$ coefficients are listed in Table 2. 
Table 2. The parameters of the Redlich-Kister polynomial for the excess free energy in the stishovite-rutile solid solution

\begin{tabular}{|c|c|c|c|}
\hline$n$ & $\begin{array}{c}A_{n} \\
(\mathrm{~kJ})\end{array}$ & $\begin{array}{c}B_{n} \cdot 10^{3} \\
(\mathrm{~kJ} / \mathrm{K} / \mathrm{mol})\end{array}$ & $\begin{array}{c}C_{n} \cdot 10^{6} \\
\left(\mathrm{~kJ} / \mathrm{K}^{2} / \mathrm{mol}\right)\end{array}$ \\
\hline 0 & 9.95 & 25.99 & 1.70 \\
\hline 1 & -11.36 & 6.66 & 2.08 \\
\hline 2 & 1.16 & 17.56 & 3.83 \\
\hline 3 & 3.35 & -4.78 & 1.14 \\
\hline 4 & 12.27 & 3.31 & 3.13 \\
\hline
\end{tabular}

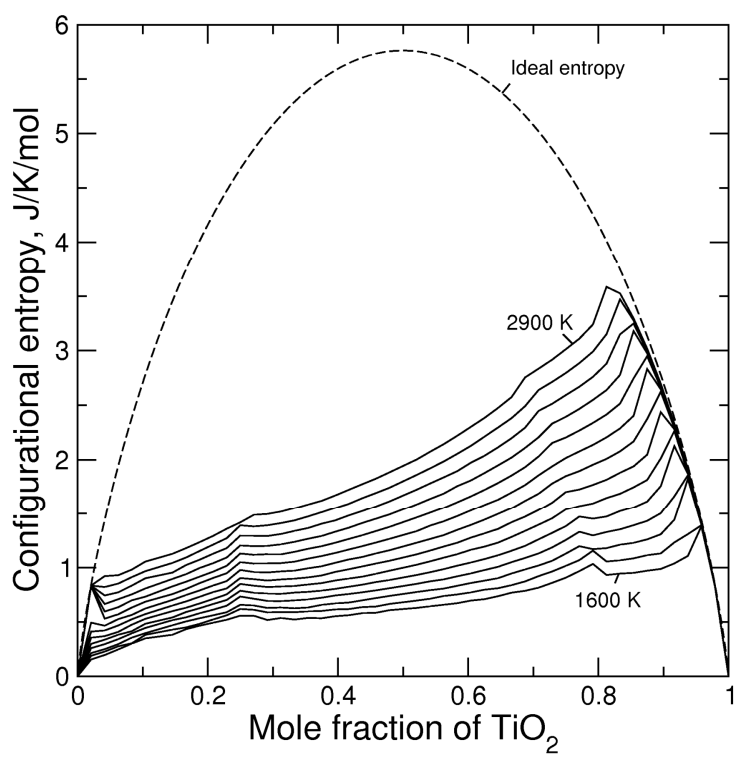

Fig. 4. The configurational entropy calculated with the equation $S=(H-G) / T$ using the calculated enthalpies and the free energies of mixing.

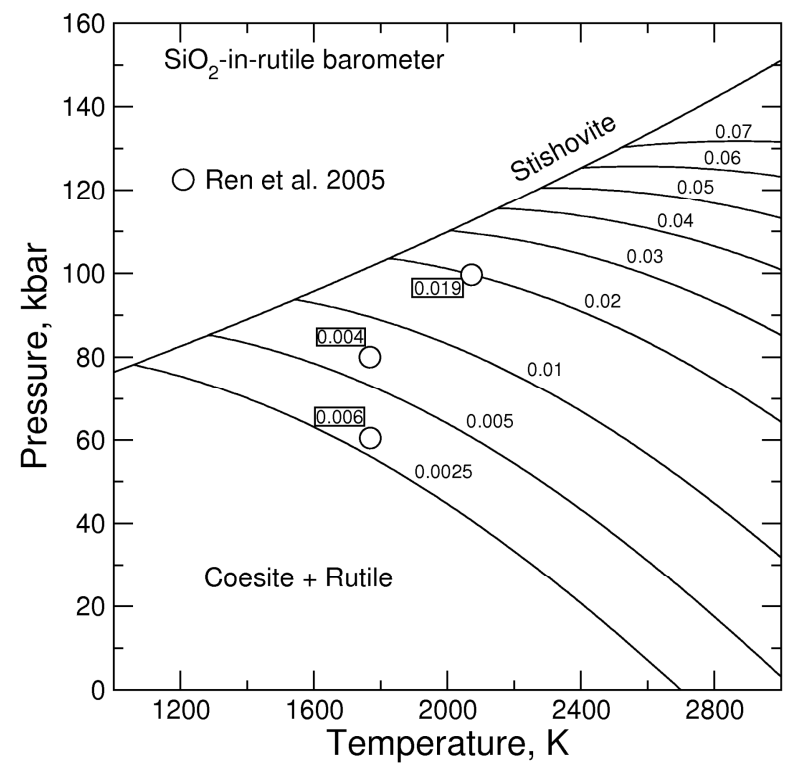

Fig. 5. The isopleths of $\mathrm{SiO}_{2}$ mole fraction in rutile in equilibrium with coesite predicted using the presently derived activity-composition model. The experimental results of Ren et al. (2005) are also shown. The thermodynamic properties of coesite and stishovite are from the data base of Holland and Powell (1998).

The derived activities of the $\mathrm{SiO}_{2}$ component were used to calculate the stishovite(rutile)-coesite equilibrium. The isopleths of $\mathrm{SiO}_{2}$ concentration in rutile in equilibrium with coesite (Fig. 5) are cut with the coesite/stishovite transition boundary where rutiles become saturated with $\mathrm{SiO}_{2}\left(a_{\mathrm{SiO}_{2}}=1\right)$. Our results are in good agreement with the experimental data of Ren et al. (2005).

Our calculations predict that the Si-in-rutile barometry would be very efficient at temperatures higher than $2000 \mathrm{~K}$, where the Si-concentrations reach first mole $\%$. For lower temperatures, a very sensitive local analytical technique would be needed. The method relies on the assumption that coesite is a pure phase and is applicable only in the cases where rutiles have not been transformed into the high pressure $\alpha \mathrm{PbO}_{2}$ type structure. Further studies of the thermodynamics of $\mathrm{SiO}_{2}-\mathrm{TiO}_{2}$ mixing in coesite and $\alpha \mathrm{PbO}_{2}$ structures would be useful to clarify possible errors due to violations of these assumptions.

We thank Dr. Yufeng Ren for the access to details of his experimental study.

\section{References}

Clark, S.J., Segall, M.D., Pickard, C.J., Hasnip, P.J., Probert, M.I.J., Refson, K., Payne, M.C. (2005) First principles methods using CASTEP. Z. Kristallogr., 200, 567-57.

Holland, T.J.B., Powell, R. (1998). An internallyconsistent thermodynamic data set for phases of petrological interest. J Metamorph Geol., 16, 309-343.

Kaminsky, F. V., Zakharchenko, O. D., Griffin, W. L., Channer, D. M. de R. \& Khachatrayan-Blinova, G. K. (2000) Diamond from the Guaniamo area, Venezuela. The Canadian Mineralogist, 38, 1347-1370.

Kresse, G., Hafner, J. (1994) Norm-conserving and ultrasoft pseudopotentials for first-row and transition elements. J. Phys: Condens. Matt., 6, 8245-8257.

Ren, Y., Fei, Y., Yang, J., Bai, W.J., Xu, Z.Q. (2005) $\mathrm{SiO}_{2}$ solubility in rutile at high pressure and temperature. American Geophysical Union, Fall Meeting 2005, abstract \#V51E-07.

Vinograd, V.L., Sluiter, M.H.F., Winkler, B. (2008) Prediction of the effective pairwise interactions in binary solid solutions from the enthalpies of single- and double-defect structures: I. Calculations based on empirical force-fields. Geochimica et Cosmochimica Acta (submitted).

Warren, M.C., Dove, M.T., Myers, E.R., Bosenick, A., Palin, E.J., Sainz-Diaz, C.I., Guiton, B.S. (2001) Monte Carlo methods for the study of cation ordering in minerals. Mineralogical Magazine, 65, 221-248.

Yang, J., Bai, W., Fang, Q., Yan, B., Shi, N., Ma, Zh., Dai, M., Xiong, M. (2003) Silicon-rutile - An ultra-high pressure (UHP) mineral from an ophiolite. Progress in Natural Science, 13, 528531. 
